\title{
FTIR Hyperspectral Images of Microscopic Droplets of Splattered Blood
}

Scott W. Huffman ${ }^{1}$, Kara B. Lukasiewicz ${ }^{2}$ and Chris W. Brown

University of Rhode Island cbrown@chm.uri.edu

During forensic investigations, determining the time frame of a crime can be an extremely important clue for solving the case. The exact time at which a crime was committed can be especially difficult to determine when considerable time has elapsed. To improve the predictive capabilities of crime scene investigators, we have focused on using spectroscopic methods to investigate the aging of bloodstains. As part of this investigation, we used FTIR infrared hyperspectral images of microscopic blood drops to learn more about blood-surface interactions, i.e., to follow

Single $\lambda$

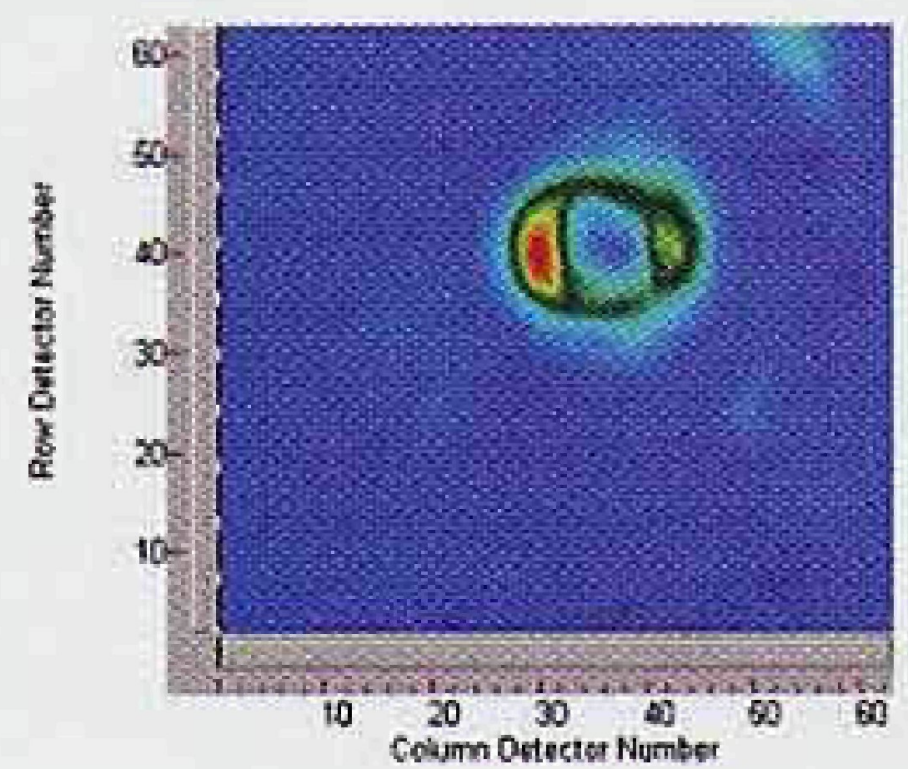

Visible Image
Total IR

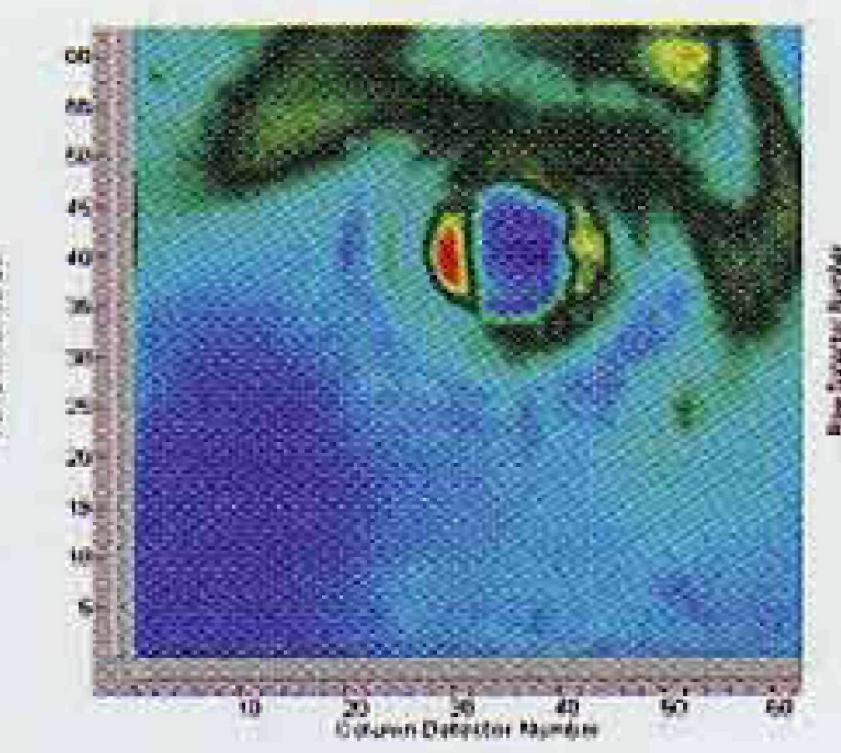

Infrared

Image

tion of IR absorbing material close to and above the ring of blood cells. The right image is formed by taking the ratio of the integrated area of the band in the $\mathrm{CH}$ stretching region of $3050-2775 \mathrm{~cm}^{-1}$ to the integrated area of the band in the $\mathrm{OH}-\mathrm{NH}$ stretching region of $3720-3050 \mathrm{~cm}^{-1}$. We refer to this ratio as the $\mathrm{CH} / \mathrm{OH}$ ratio since the major increases in the $\mathrm{OH}-\mathrm{NH}$ region are due to water; however, this region also includes the $\mathrm{NH}$ stretching absorptions. Variations in the $\mathrm{CH} / \mathrm{OH}$ ratio are best seen by comparison of the three spectra for this sample that are shown in Figure 2. Outside the ring of blood cells the spectra in the green region are very similar and there is a rather even distribution of $\mathrm{CH} / \mathrm{OH}$ ratios. The spectrum from the ring of cells exhibits a rather high $\mathrm{CH} / \mathrm{OH}$ ratio. The center of the ring of cells has a high water content and low $\mathrm{CH}$.

Three images of another microscopic blood droplet are

Figure 1. Infrared hyperspectral images of a "donut" shaped blood droplet. Left image changes in refractive index as radiation passes through the sample. Middle image - total integrate IR intensity at each pixel. Right image - ratio of $\mathrm{CH} / \mathrm{OH}$ integrated intensities.

the changes that occur on different surfaces.

FTIR hyperspectral images were measured on a Digilab Stingray FTS-6000 imaging spectrometer equipped with a Digilab UMA-500 infrared microscope and a $64 \times 64$ MCT focal plane array detector (Digilab, Randolph, MA., USA). The spectral images were obtained for $400 \times 400 \mu \mathrm{m}$ sample sections in transmission mode. Each detector pixel viewed a $6 \times 6 \mu \mathrm{m}$ spatial location and each hyperspectral image consisted of 4096 spectra. For these measurements, 80 frames acquired at $8 \mathrm{~cm}^{-1}$ resolution with a sample rate of $2.5 \mathrm{~Hz}$ were co-added. pared with vein blood samples from the same person. The blood was dropped onto BaF2 windows from a pipette held approximately 12 inches above the windows. The spectra were measured during the first hour after exposing spectrum the blood to air.

A number of the microscopic blood droplets formed a clump of cells as is shown by the left image in Figure 1. This is a false color image that is indicative of the refractive index changes as $I R$ radiation is passed through the sample and is very similar to that observed through a visible microscope. The middle image is formed by plotting the total integrated IR intensity at each pixel and shows a distribu-
$\mathrm{CH} / \mathrm{OH}$

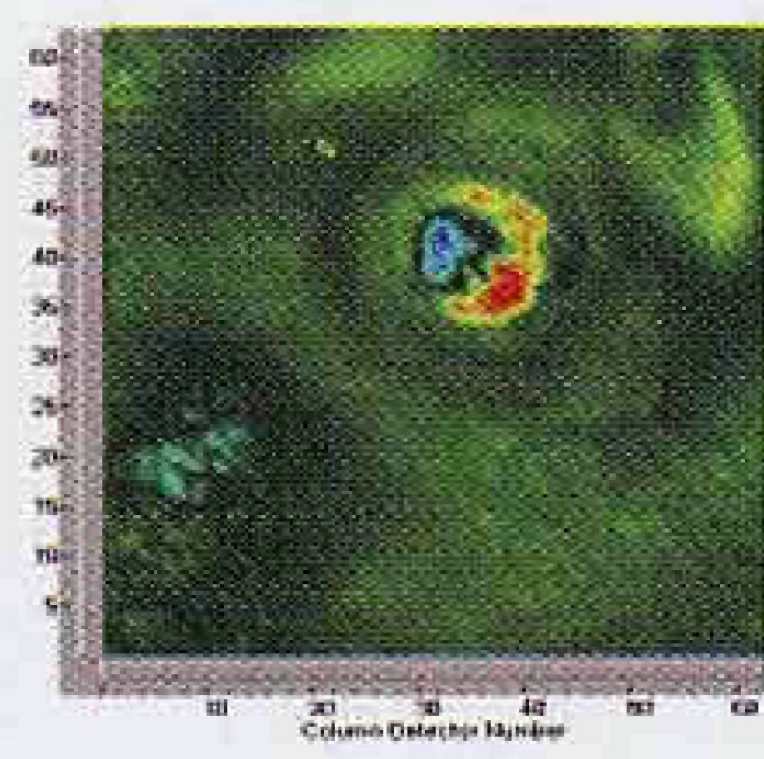

Chemical Image shown in Figure 3. The image on the left is a false color image that is indicative of the refractive index changes, and shows the blood cells piled up in a couple locations: The cells appear to be surrounded by a transparent material. The total IR image obtained by plotting the integrated spectral intensity at each pixel is shown in the middle image. In this image, the cells and material immediately surrounding the cells exhibit strong infrared absorbances, and there is a weaker absorbing material spread over the remainder of the image except for the upper right quadrant. The third image on the right is by far the most interesting. The ratios of the $\mathrm{CH} / \mathrm{OH}$ intensities (as explained above) are plotted. Regions of high $\mathrm{CH} / \mathrm{OH}$ are seen in the lower right quadrant. The absorptions outside of the cell locations are due to serum and it appears that the serum separates into regions of high $\mathrm{CH}$ and high water as shown in the lower right quadrant. This image appears very similar to a river delta in that proteins separate from water and are deposited as the serum flows away from the cells. In a separate experiment, it was found that upon complete drying, the material in this

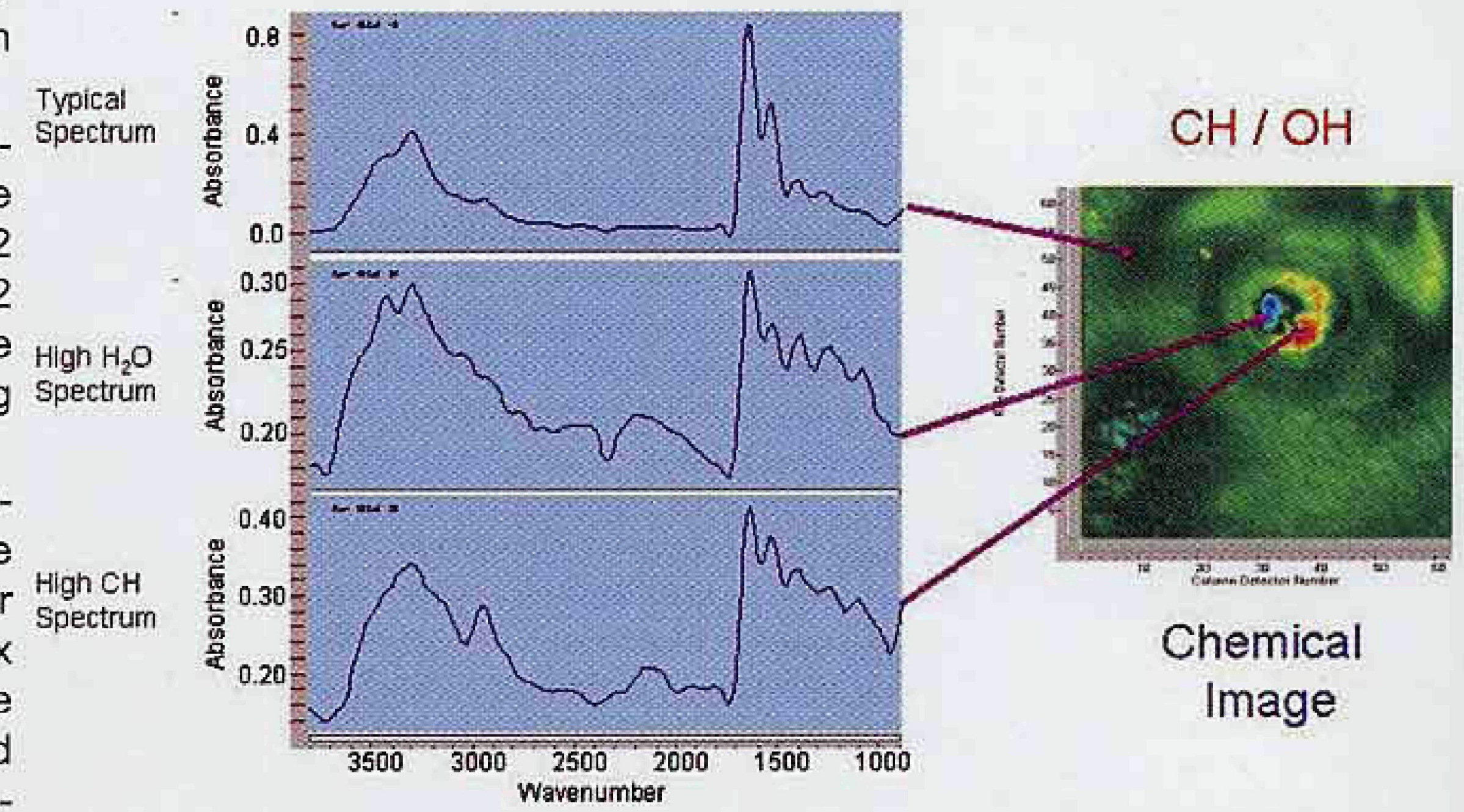

Figure 2. Infrared spectra from three different regions in the hyperspectral image shown in Figure 1. 


\section{DIGILAB FASTIMAGEIR ${ }^{\text {m }}$ \\ THE WORLD'S MOST AFFORDABLE FTIR IMAGING SYSTEM DELIVERS FLAWLESS IMAGES AT AMAZING SPEED}

Imagine the power to see images at an unprecedented level of detail.

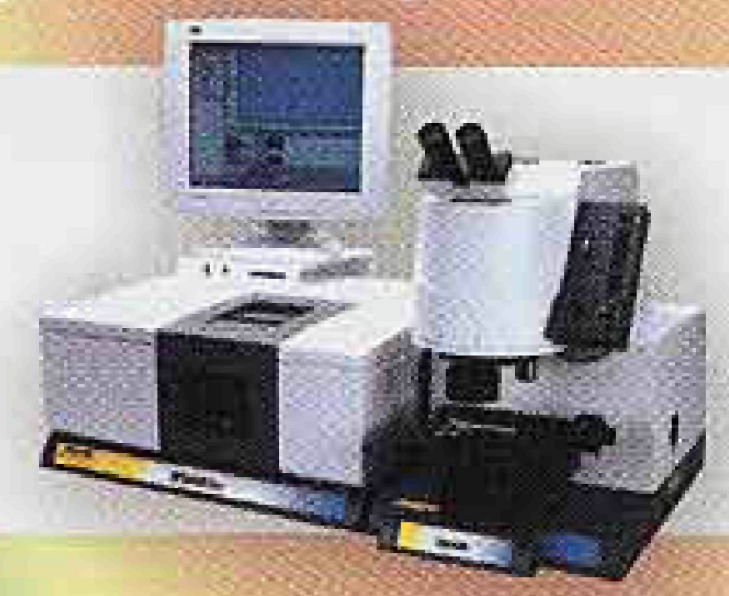

- Up to 4,096 spectra collected simultaneously across a sample

- Incredibly reliable and easy-to-use

- World's price/performance leader

- 2-D spatial information in seconds

That's performance impossible to achieve through normal or fast mapping. Own a solution that generates the best analytical answers in record time. Digilab offers a full line of FTIR spectrometer systems for quality control and research applications.

Digilab: Spectroscopy's Leading Light ${ }^{\text {mi }}$

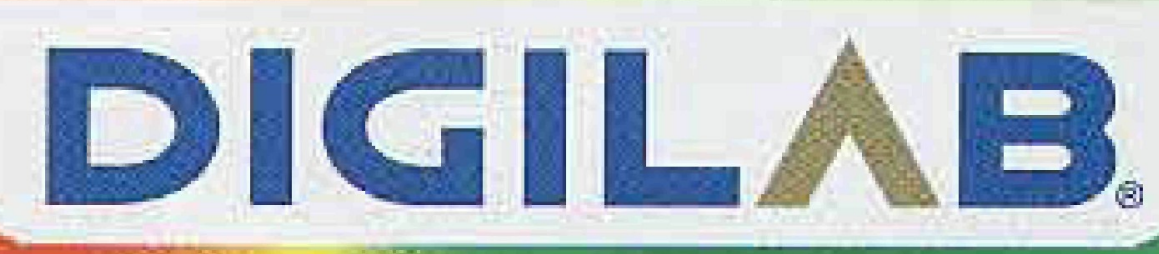


Single $\lambda$

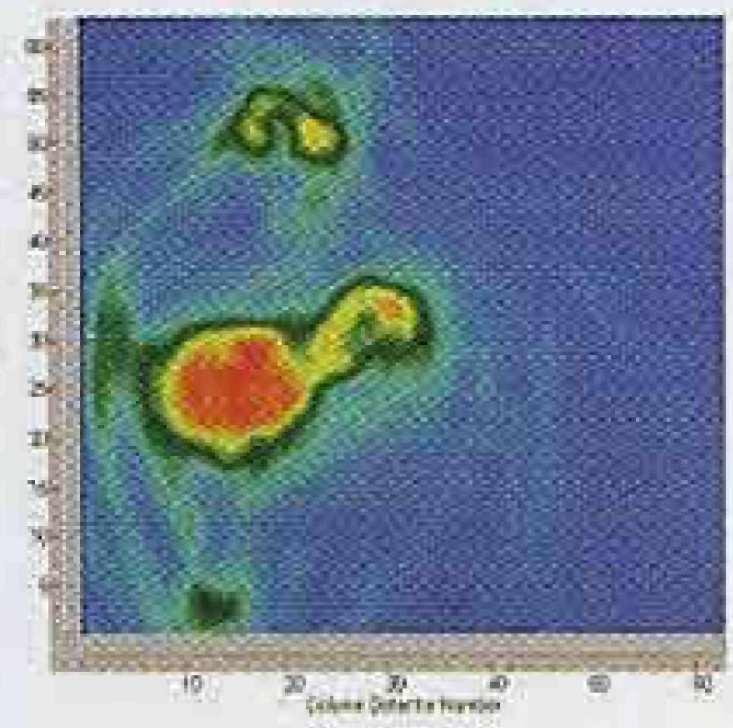

Visible Image
Total IR

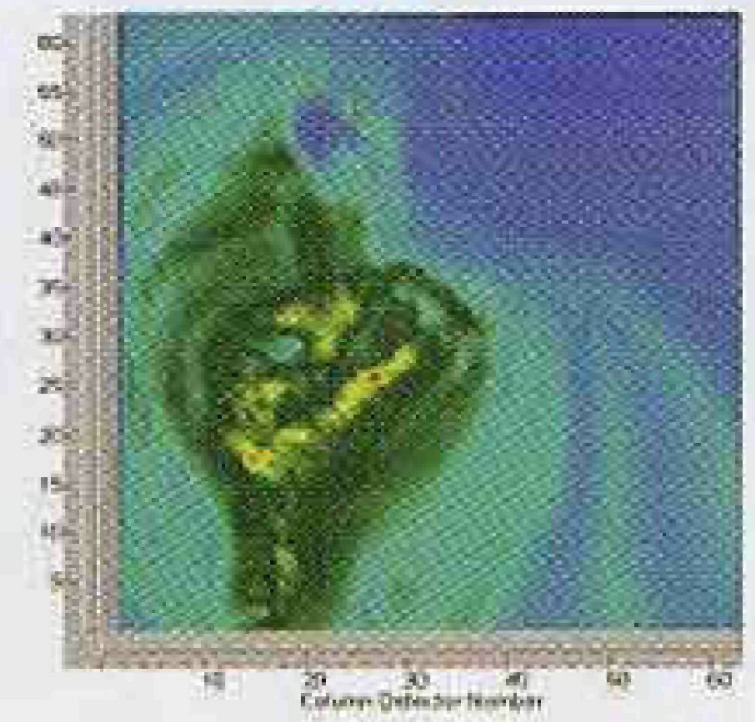

Infrared

Image
$\mathrm{CH} / \mathrm{OH}$

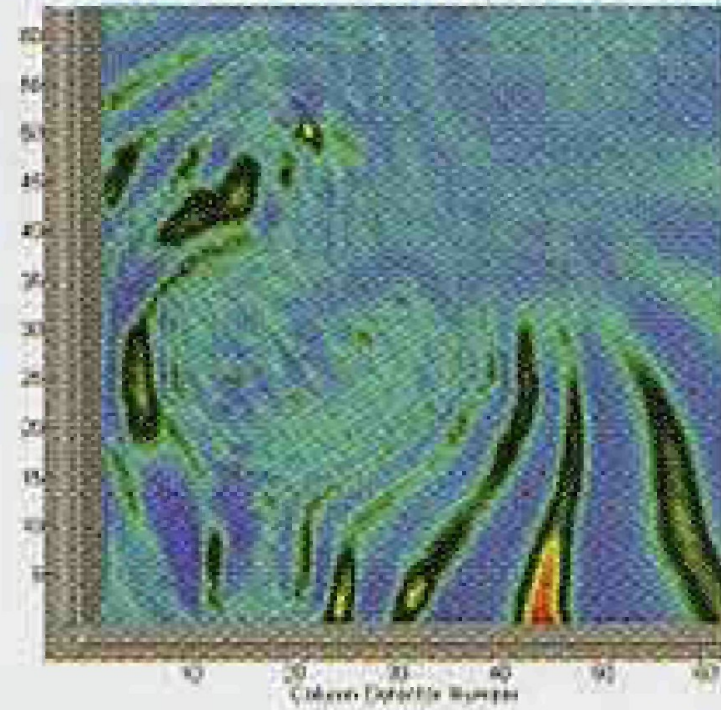

Chemical Image

Figure 3.Infrared hyperspectral image of blood droplets. Left image - refractive index changes. Middle image - total integrated IR. Right image - ratio of $\mathrm{CH} / \mathrm{OH}$ integrated intensities.

lower right quadrant exhibit cracks in the areas of higher water content, whereas the areas high $\mathrm{CH}$ (proteins and lipids) remained intact.

Similar types of hyperspectral IR images have been obtained from microscopic blood drops on fabrics and other porous materials. The observed changes are different from those observed here, since water is often preferentially removed by the substrate and flow of the serum away form the blood cells cannot be characterized. However, IR images have clearly demonstrated that there is a separation of cells and serum on non-porous surfaces. Several donut shaped droplets were detected and, although of different sizes, the separations of components were similar to those observed for the donut shaped droplet in Figures 1 and 2. The pat-

terns shown in the right image in Figure 3 are quite interesting and would not have been detected without use of infrared images.

During the investigation using IR-spectra to predict the age of a bloodstain, we discovered that vein and capillary stains exhibit different IR-spectra. Later, it was found that visual images of vein and capillary blood are grossly different when microscopic samples are viewed. Currently, we are measuring IR hyperspectral images of capillary blood samples using the Digilab FTS-7000 Stingray equipped with a Lancer focal plane array detector. This second generation instrument acquires data faster with an improved signal to noise ratio. The spectral images of the capillary blood samples will be compared with the IR images of the vein samples discussed here. These comparisons will be the subject of a future report.

\section{Endnotes}

1 Current address: National Institutes of Health, Bethesda, MD

2 Current address: Mayo Clinic, Rochester, MN.

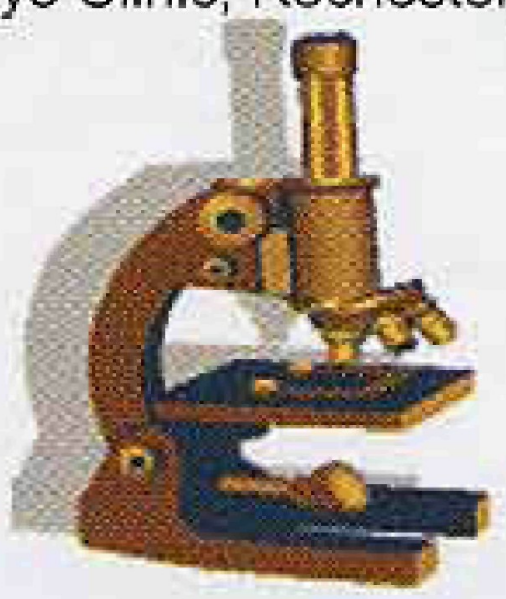

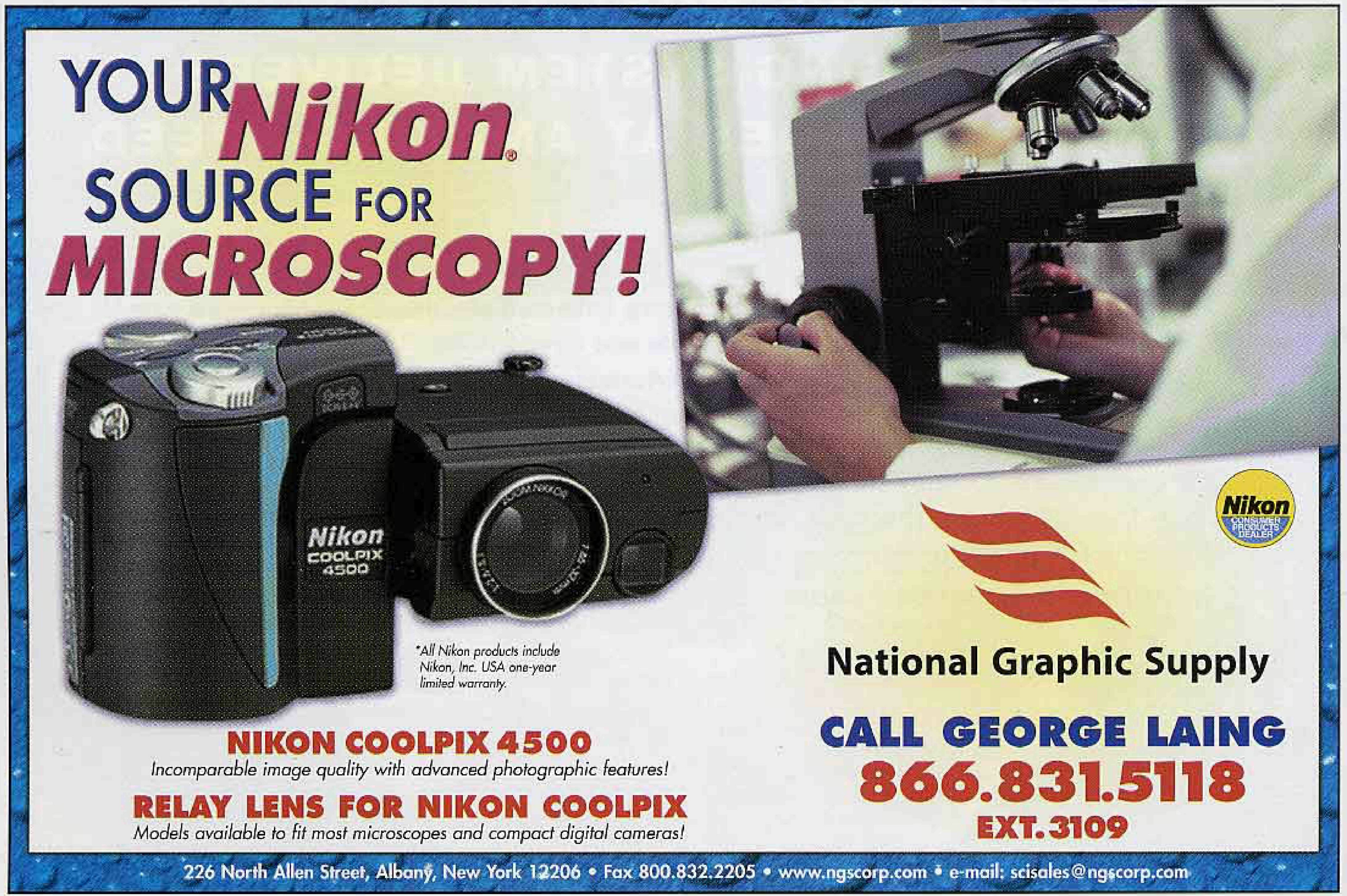




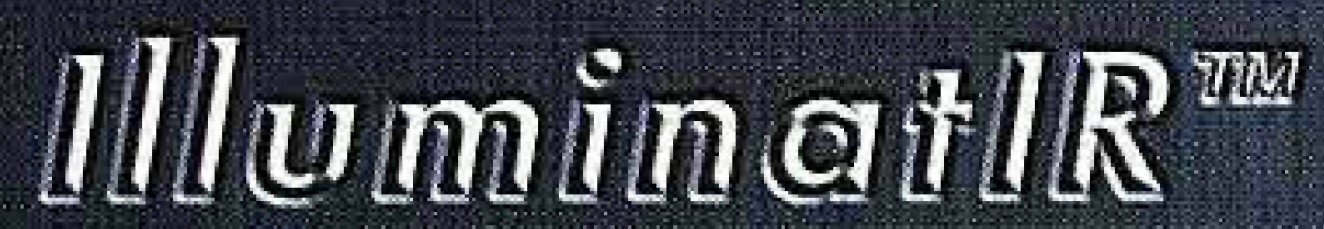

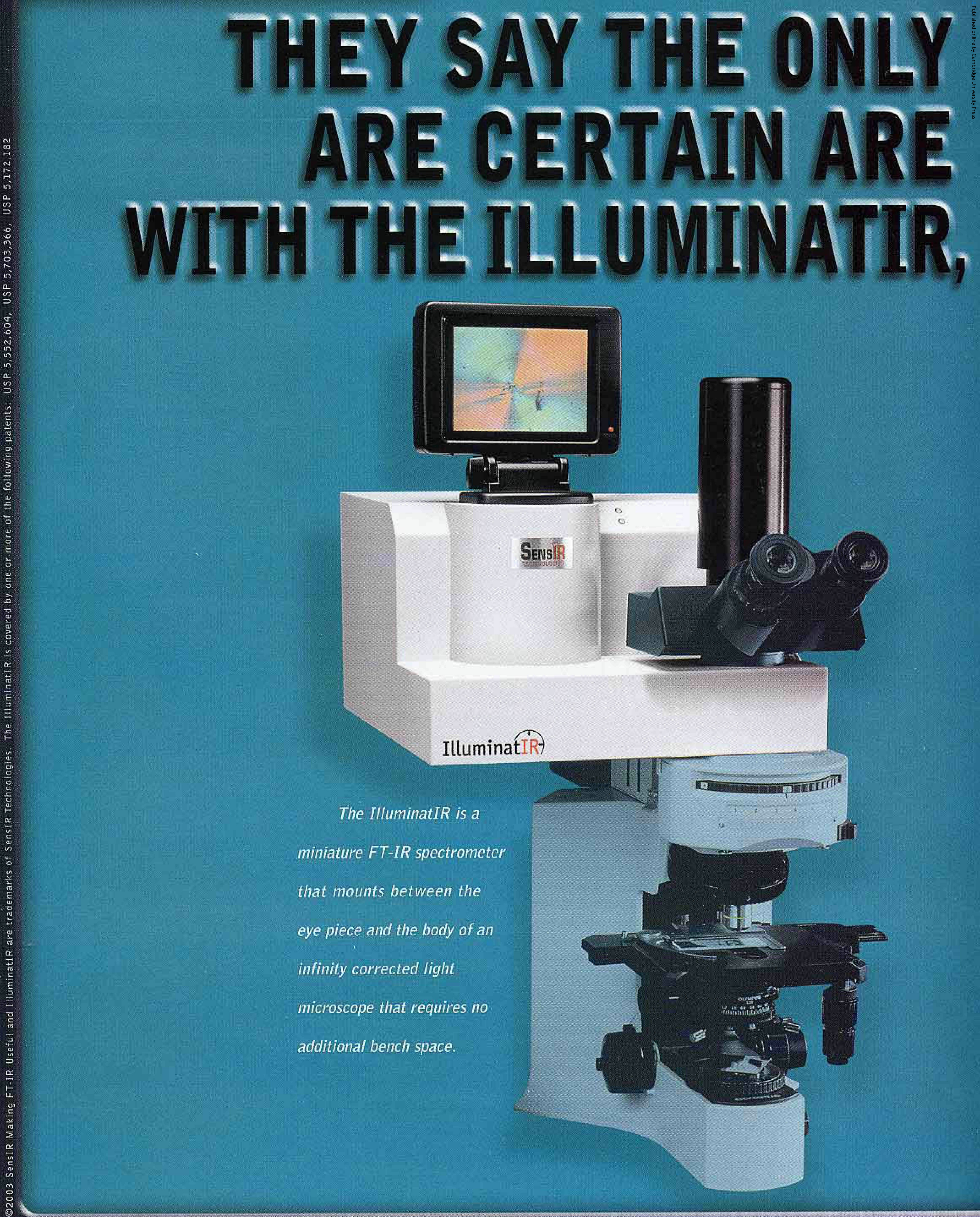




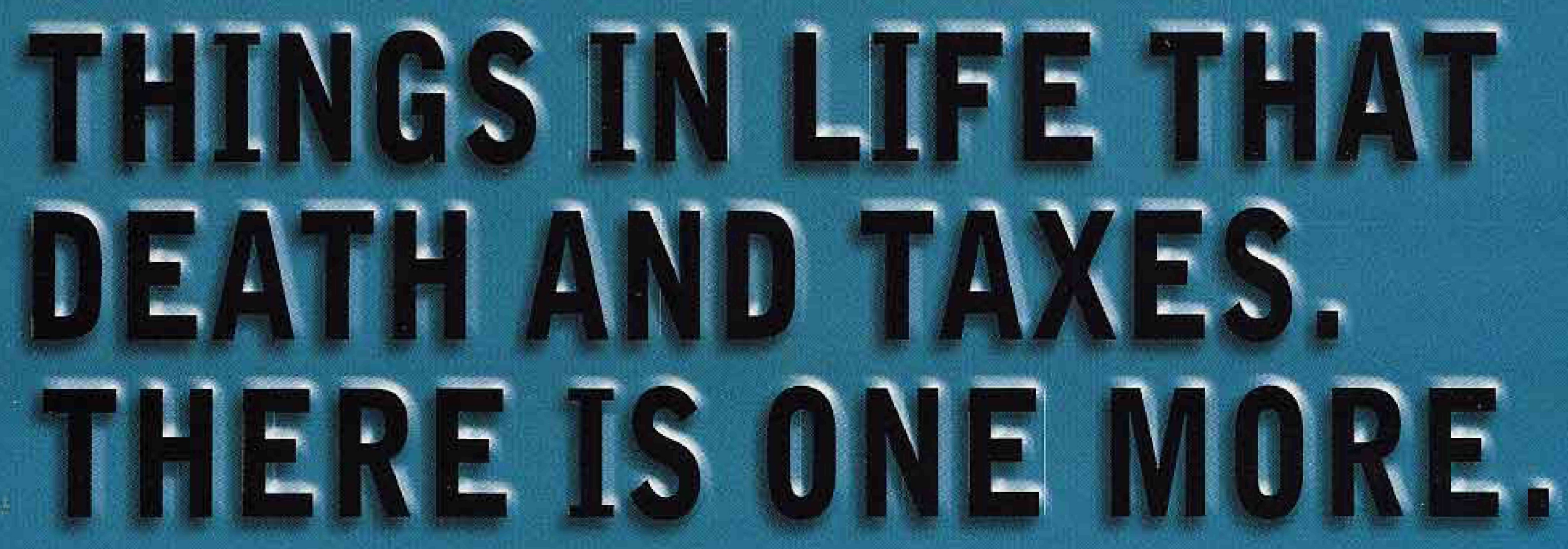

\section{IlluminatIR adds the power of FT-IR analysis to your light microscope}

Introducing the IlluminatIR, the only miniaturized FT-IR system that attaches directly to your favorite light microscope. Add the power of molecular analysis to your visual interpretation for the most accurate and objective results.
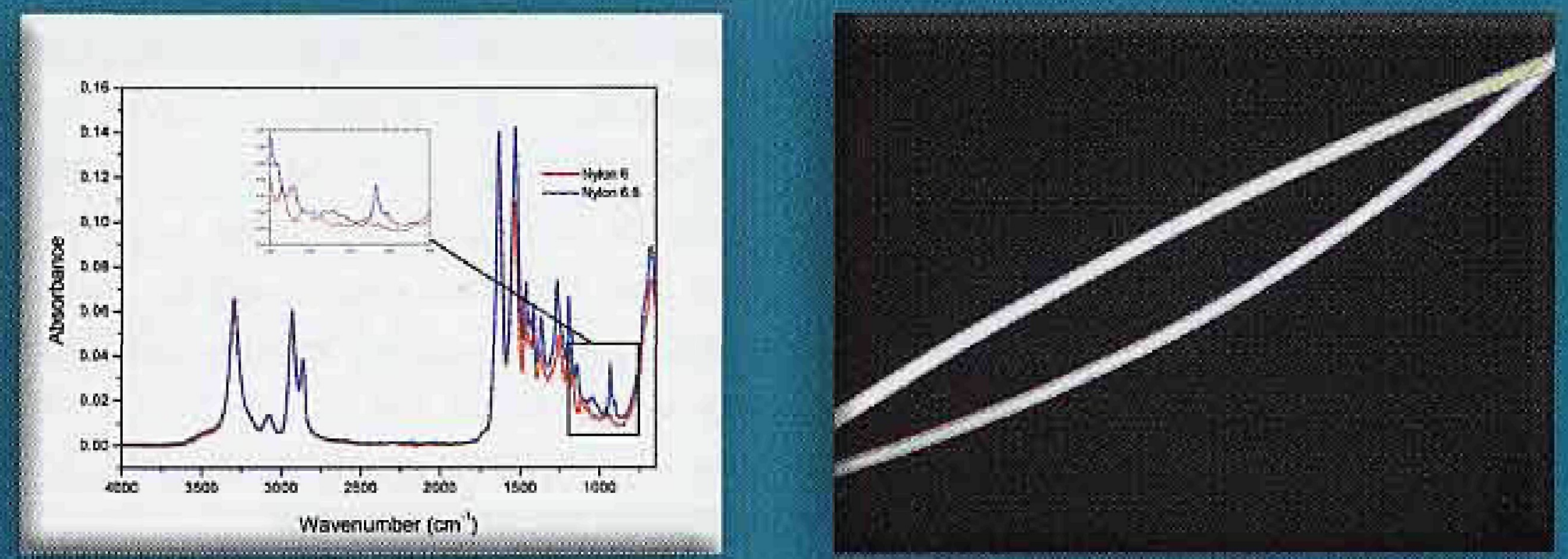

When samples are visually identical, FT-IR mitroscopy can show you the difference.

Visually these two nylon fibers are identical. However, when $F T-I R$ analysis is rum on each of the fibers and their spectra is overlaid the difference in the two fibers becomes cleat The top fiber was identified as Mylon 6 and the bottom fiber to be Nyion 6,6 .

Only IlluminatIR:

- provides high quality infrared data and superior visual quality

- easily attaches to infinity corrected microscopes

- maintains all the capabilities of your microscope,

i.e. polarization, fluorescence, Nomarski or image analysis, etc.

- can run FT-IR analysis simply with the turn of an objective - no need to move the sample

- adds these capabilities without taking up additional bench space

- very easy to use and maintain

The IIluminatIR: it will change the way microscopes are used forever.

\section{MAKE YOUR OWN OBJECTIVE OPINION. \\ Call us to arrange a \\ free demonstration of this breakthrough technology. \\ 1-888-473-6747 \\ Or visit our website www.sensir.com/objective

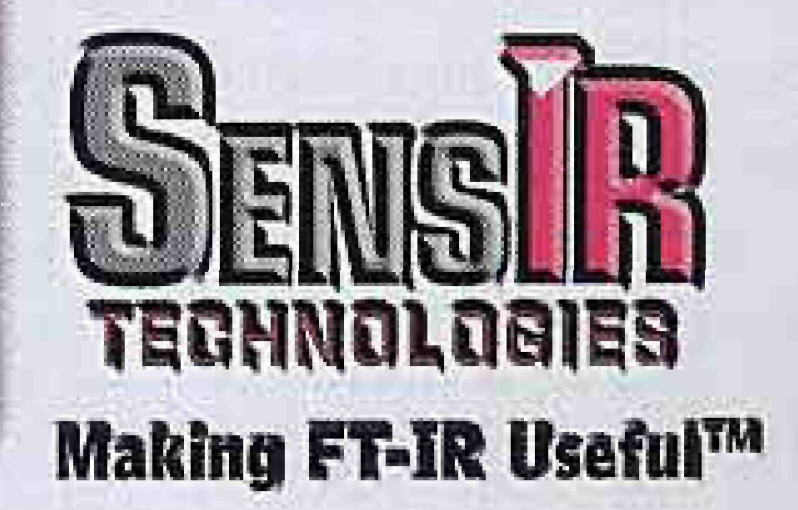

